\title{
Double Meningioma: A Case of Two Fibrous Meningiomas Coexisting Isolatedly in Meningothelial Meningioma
}

\author{
Kagemichi NAGAO, ${ }^{1}$ Katsumi SAKATA, ${ }^{1}$ Takashi KAWASAKI, ${ }^{1}$ \\ Hiroshi MANAKA, ${ }^{1}$ Koichi URAmaru, ${ }^{1}$ Tetsuya Yamamoto, ${ }^{2}$ \\ and Makoto SHIBUYA ${ }^{3}$ \\ ${ }^{1}$ Department of Neurosurgery, Yokohama City University Medical Center, Yokohama, \\ Kanagawa, Japan \\ ${ }^{2}$ Department of Neurosurgery, Yokohama City University School of Medicine, Yokohama, \\ Kanagawa, Japan \\ ${ }^{3}$ Central Laboratory, Tokyo Medical University Hachioji Medical Center, Hachioji, Tokyo, Japan
}

\begin{abstract}
Meningiomas are the most common intracranial primary neoplasm in adults, and show various histological subtypes, indicating heterogeneous clinical and molecular genetic characteristics. Different subtypes of meningioma coexisting independently within the main tumor of another different subtype is a quite rare clinical situation. A 69-year-old woman presented with a severalyear history of dizziness as a non-specific complaint. Magnetic resonance imaging (MRI) revealed an extra-axial mass lesion in the left parieto-occipital region including two well-demarcated, round mass components. Total resection was performed via left parieto-occipital craniotomy. Two white masses were identified within the main tumor, with neither showing dural attachments. Pathological findings showed the main mass represented meningothelial meningioma and the demarcated mass lesions were both fibrous meningiomas. No transitional features existed between these subtypes. No differences in genetic characteristics were evident between subtypes of meningioma. We have described, apparently for the first time, a case of two fibrous meningiomas coexisting in an isolated manner in meningothelial meningioma with the similar molecular genetic profile.
\end{abstract}

Keywords: fibrous meningioma, meningothelial meningioma, coexisting tumor

\section{Introduction}

Meningiomas arise from arachnoid cap cells, and are one of the most frequently encountered intracranial tumors. This pathology accounts for $20-36 \%$ of all primary tumors with an annual incidence of up to 1.8-13 per 100000 population. ${ }^{1)}$ These are categorized by the World Health Organization (WHO) into three grades with 15 histological subtypes, indicating heterogeneous clinical and molecular genetic characteristics. According to a recent study, various types of mutation have been discovered in

Received May 14, 2020; Accepted September 3, 2020

Copyright $\subseteq 2021$ by The Japan Neurosurgical Society This work is licensed under a Creative Commons AttributionNonCommercial-NoDerivatives International License. non-neurofibromatosis type 2 (NF2) meningioma, correlating with anatomical tumor location and histological subtypes. ${ }^{2)}$ Although the majority of meningiomas are single lesions, multiple lesions are present in up to $10 \%$ of cases. ${ }^{3)}$ Multiple meningiomas are defined as the presence of two or more spatially separated synchronous or metachronous lesions, and these can also be sporadic, radiation-induced or familial. The two familial syndromes commonly associated with the disorder are NF2 and familial meningiomatosis. Several authors have reported the same patient showing meningiomas of differing histology and grade. ${ }^{4-6)}$ Those cases of multiple meningiomas involved different or neighboring sites. No reports appear to have described isolated meningioma of one subtype coexisting within meningioma of a different subtype. We report herein the case of 
a patient with fibrous meningioma distinctly coexisting within meningothelial meningioma.

\section{Case Presentation}

A 69-year-old woman presented with a several-year history of dizziness as a non-specific complaint. She was referred to our hospital after magnetic resonance imaging (MRI) in a local hospital revealed a mass lesion in the left parieto-occipital region. No neurological findings were evident on admission. Preoperative MRI revealed an extra-axial mass lesion in the left parieto-occipital region, appearing to contain two well-demarcated round mass components (Fig. 1). Diffusion-weighted MRI showed hypointense round masses within a hyperintense main tumor (Fig. 1A). T1-weighted imaging revealed isointense round masses within the main tumor (Fig. 1B). T2-weighted imaging demonstrated two low-intensity, round masses and a high-intensity main tumor (Fig. 1C). Contrast-enhanced MRI showed enhancement of both components, with higher enhancement of the main tumor compared to the round masses (Fig. 1D). As the tumor diameter was up to $7 \mathrm{~cm}$ on MRI and preoperative carotid angiography revealed a massive tumor stain fed by the left middle meningeal artery, we planned preoperative embolization followed by surgical extirpation.

Total resection was performed via a left parieto-occipital craniotomy after preoperative embolization. Simpson grade I removal was achieved. Two white masses were identified within the main tumor, with neither showing dural attachment (Fig. 2). The postoperative course was uneventful. MRI one year after surgery showed no recurrence (Fig. 3).

Microscopic examination of a specimen from the main tumor showed typical meningothelial meningioma (Fig. 4A) while a specimen from one of the buried mass lesion revealed typical fibrous meningioma (Fig. 4B). The margins of both meningioma subtypes were clear, with no evidence of transitional mixtures of either subtype of meningioma (Fig. 4C and 4D). MIB-1 index was less than 3\% for both tumor components. Comparing the two tissues, amounts of collagen fibers differed markedly, as did cell density. In addition, findings such as necrosis, degeneration, and embolic materials in the vessels were observed in part of the meningothelial component, probably due to the effects of preoperative embolization.

Molecular genetics were analyzed for both meningothelial and fibrous subtypes by fluorescent in situ hybridization (FISH) and Sanger sequencing. ${ }^{7)}$ No mutations were seen for NF2. In addition, $A K T 1$, KFL4, SMO, TRAF7, and PI3K were all wild type
(WT). Meningothelial meningioma showed C195G mutation in the TERT promotor while the fibrous meningioma was WT in the TERT promotor.

\section{Abbreviations \\ neurofibromatosis $(N F)$, alpha serine-threonine protein kinase $(A K T)$, Kruppel-like factor $(K L F)$, smoothened frizzled class receptor (SMO), tumor necrosis factor receptor-associated factor (TRAF), phosphatidylinositol-3-kinase (PI3K), telomerase reverse transcriptase (TERT)}

\section{Discussion}

This paper reports a case of two isolated fibrous meningiomas coexisting within meningothelial meningioma. To the best of our knowledge, the situation of meningiomas presented here has not been reported previously.

Meningiomas are common brain tumors, accounting for approximately one-third of all primary brain tumors in adults. ${ }^{3)}$ In an autopsy series, incidental meningioma was identified in $2.3 \%{ }^{8)}$ Multiple meningiomas occur in up to $10 \%$ of meningiomas, but most exist as spatially separated lesions. Surprisingly, the majority of multiple meningiomas have shown different histological subtypes and even different grades. ${ }^{3,4,9)}$

The origins and pathogenic mechanisms underlying multiple meningiomas remain controversial. One possibility is that different tumors have different origins, with multiplicity not a result of cell migration through the subarachnoid space. ${ }^{9)}$ On the other hand, several studies have supported the theory of clonal spread from a single tumor. ${ }^{4,10)}$ The origin of multiple meningiomas is probably intriguing and not solely explicable on the basis of microscopic appearance.

Liu et al. ${ }^{5)}$ reported a case of meningioma with different histological grades in the same patient. The intradural component showed fibrous meningioma of WHO grade I while an epidural component invading to the skull represented atypical meningioma of WHO grade II. Yan et al. ${ }^{11)}$ reported a case of solitary fibrous tumor concurrent with meningioma in the same site. Another rare phenomenon that was reported was metastasis of breast carcinoma to meningioma. ${ }^{12)}$ Pathological examination in our case showed two fibrous meningiomas distinctly coexisting within the main tumor of meningothelial meningioma. Both fibrous meningiomas were buried separately and existed without any dural attachments within the main tumor. Transitional meningioma as one of the common variants of meningioma contains meningothelial and fibrous patterns as well as transitional 


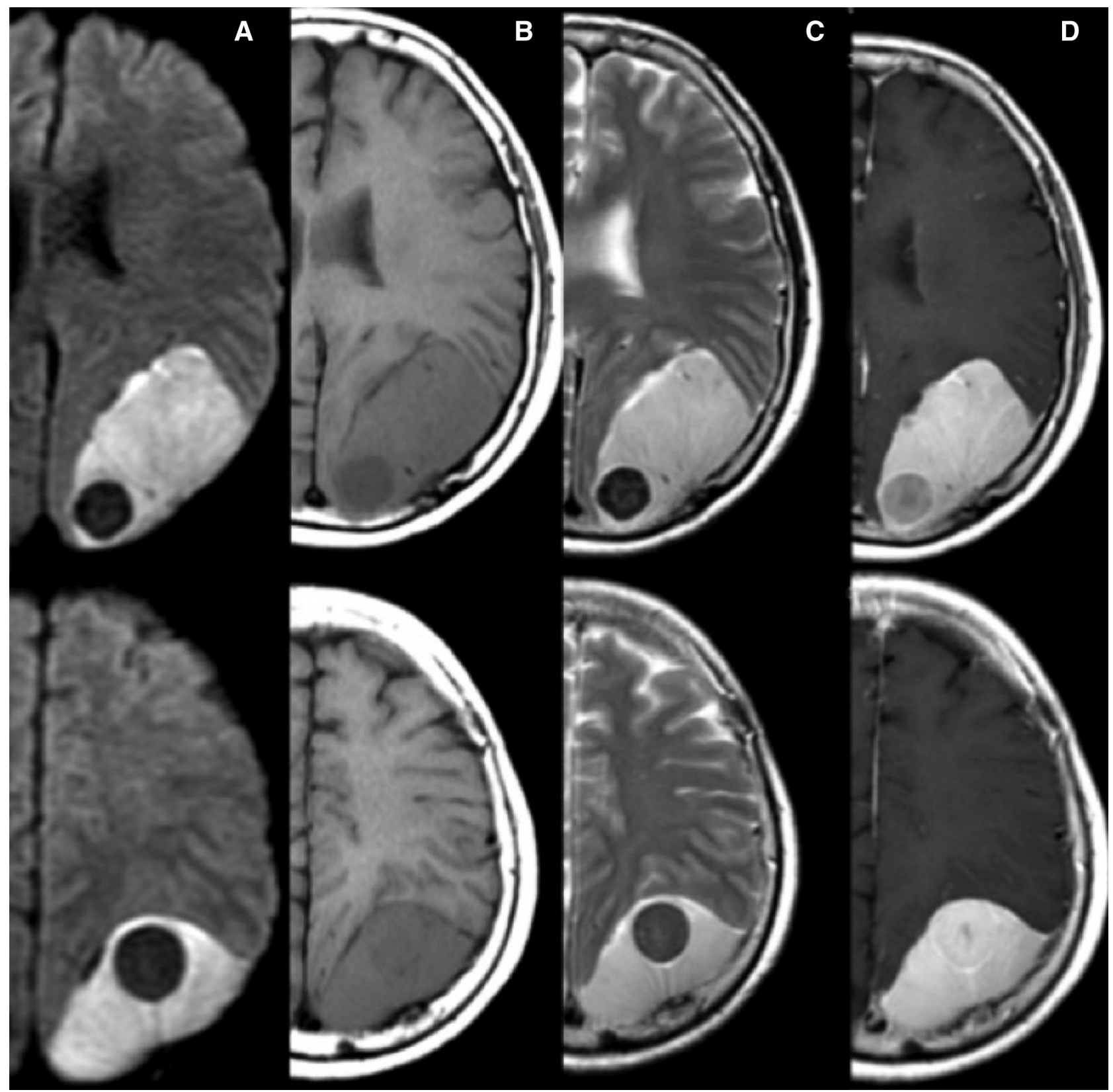

Fig. 1 Axial preoperative MRI reveals an extra-axial mass lesion in the left parieto-occipital region, including two well-demarcated round mass components. (A) Diffusion-weighted imaging shows low-intensity, round masses and a high-intensity main tumor. (B) T1-weighted imaging shows isointense findings for both round masses and the main tumor. (C) T2-weighted imaging shows hypointense round masses and a hyperintense main tumor. (D) Contrast-enhanced MRI shows enhancing effects on both components, with higher enhancement of the main tumor compared to the round masses. MRI: magnetic resonance imaging.

features. In this case, the meningothelial component and fibrous component existed not transitionally, but as distinctly separate features according to pathological examination. This was the reason the pathological diagnosis differed from transitional meningioma. Imaging studies also revealed two clearly demarcated, round mass lesions within the main component of the tumor. These imaging findings also indicated this meningioma was completely different from transitional meningioma, because two isolated tumors of a different type existed with a clear margin between each other and without transitional features. This type of meningioma does not appear to have been reported previously. 


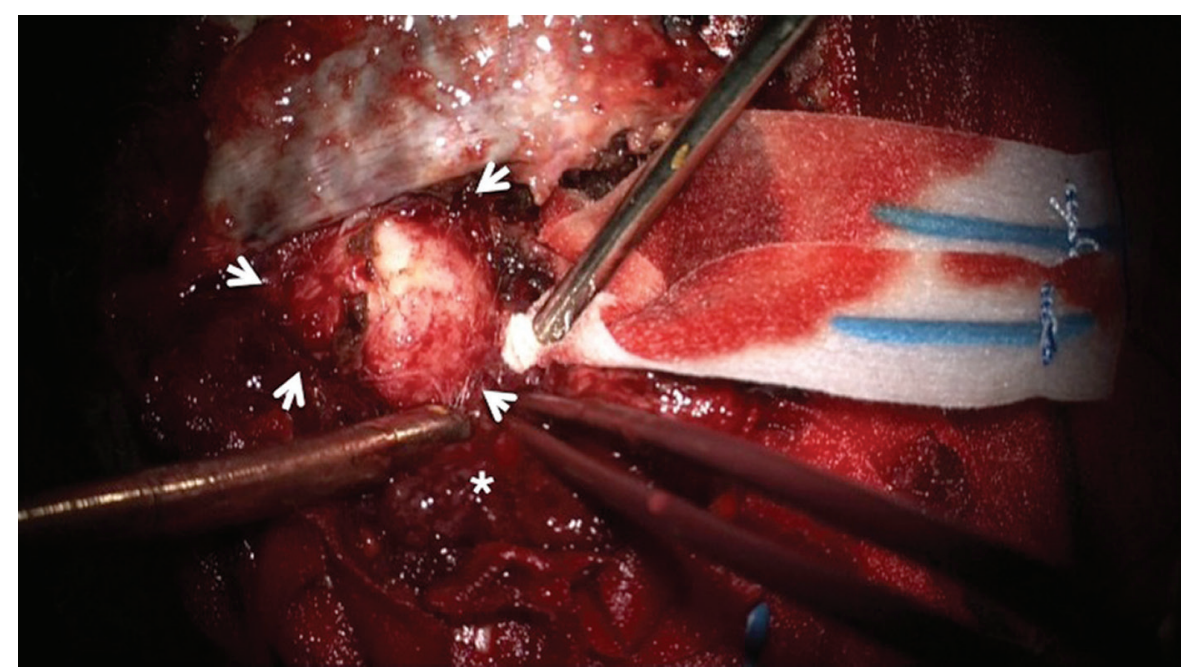

Fig. 2 Intraoperative photo shows a white, isolated round mass (short arrows) buried in the reddish main tumor $\left({ }^{*}\right)$, which has no dural attachment.

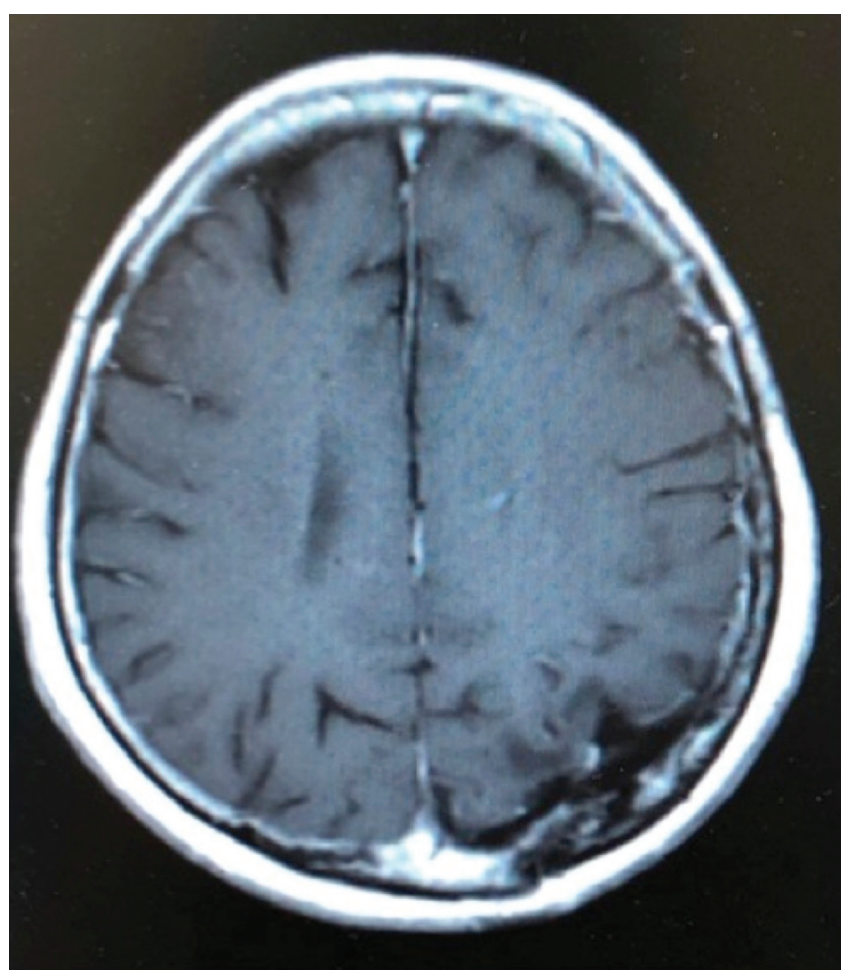

Fig. 3 Postoperative MRI at 1 year after surgery demonstrates total removal of the tumor. MRI: magnetic resonance imaging.

According to recent genome-wide genotyping and exome-sequencing studies, novel mutations have recently been discovered in non-NF2 meningiomas. ${ }^{2,13)}$ Although the most common mutated gene is NF2, newly discovered mutations in TRAF7 (24\%; encoding a proapoptotic E3 ubiquitin ligase), AKT1 (10-15\%; encoding a key effecter of PI3K signaling), KLF4 (10\%; encoding a $\mathrm{C} 2 \mathrm{H} 2$ zinc finger motif), and $S M O$ (3-5\%; encoding a negative regulator of the Hedgehog pathway) were mutually exclusive of NF2 mutations. In addition, such mutation types appear to correlate with anatomical tumor location and histological subtypes. ${ }^{2}$ Relationships between these gene mutations and subtypes of meningioma have been reported. For example, secretory meningiomas show simultaneous deletion of KLF7 and TRAF7. ${ }^{1)}$ Molecular genetic analyses in our case showed only C195G mutation in the TERT promoter in the meningothelial subtype. TERT mutation occurred in $4.7 \%$ of WHO Grade I meningiomas. ${ }^{14)}$ High-grade meningioma is reported to show the emergence of mutations in the TERT promoter. ${ }^{15)}$ On the other hand, mutation of TERT does not affect WHO classification. ${ }^{14)}$ In addition, the recurrence rate was 4.8 times higher in patients with WHO Grade I or II meningiomas with TERT mutation compared with WHO Grade III meningiomas with TERT protein WT patients. ${ }^{14)}$ TERT mutation may have some influence on the biological activity of meningiomas. No previous reports have described C195G mutation in the TERT promoter detected in this case. At the moment, the C195G mutation in the TERT promotor detected in this case could be suggested as uncertain clinicopathological significance and indicate no clinical significance. Concluding that significant differences exist in genetic profiles between these subtypes is thus difficult.

However, close imaging follow-up may be warranted for patients with meningioma showing TERT mutation. From the perspective genetic analysis, 

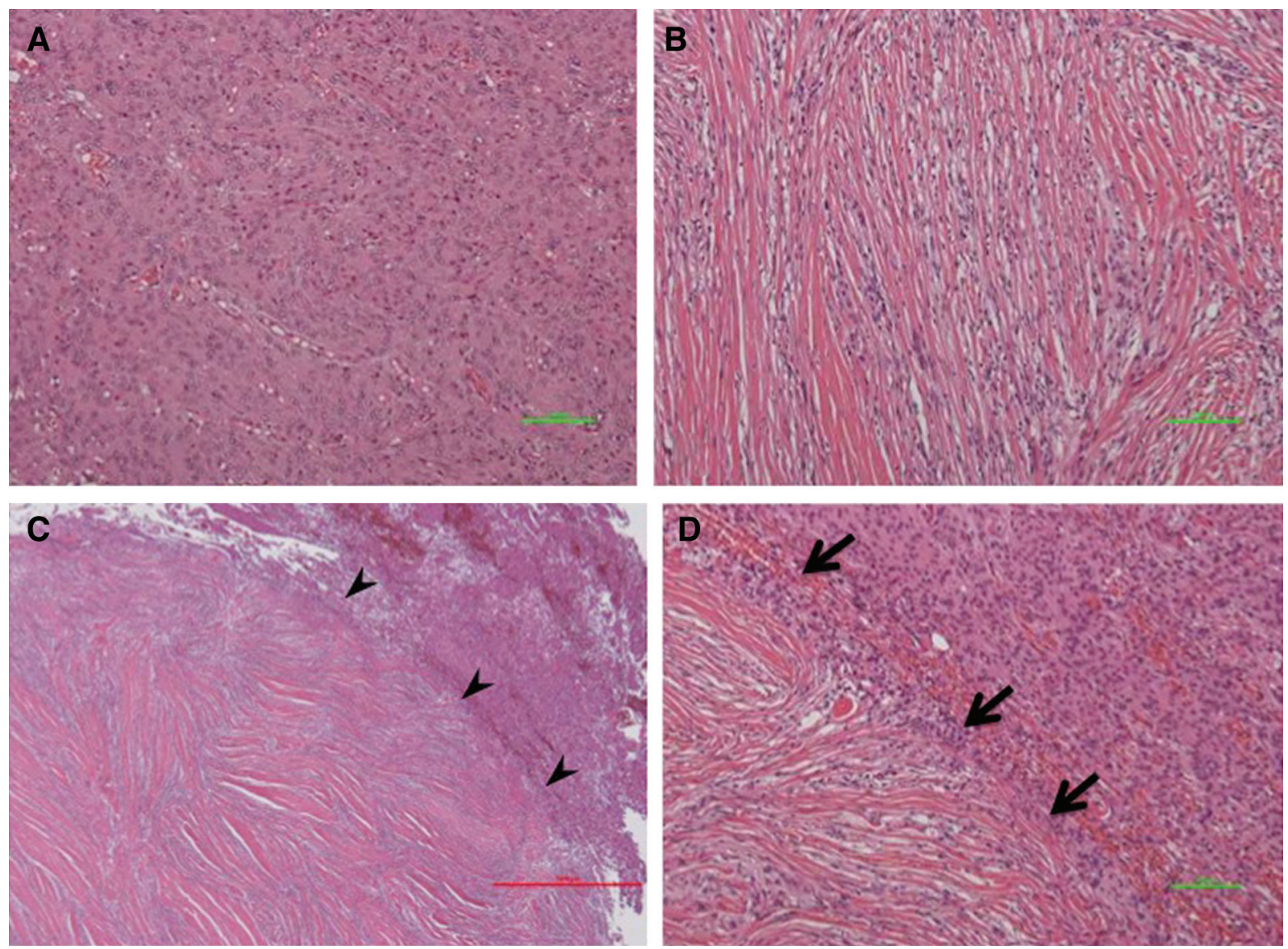

Fig. 4 Photomicrographs of pathological specimens. green bar, $100 \mu \mathrm{m}$; red bar, $1000 \mu \mathrm{m}$. (A) Specimen of the main tumor shows typical meningothelial meningioma. HE: original magnification $\times 20$. (B) A specimen of a white, round mass buried in the main tumor shows typical fibrous meningioma. HE, original magnification $\times 20$. (C, D) Specimen from the border of these components. The arrowheads (C) and the short arrows (D) indicate the border between meningothelial and fibrous subtypes. These two different subtypes of meningioma separately exist with clear margins. HE, original magnification $\times 4$ (C), $\times 20$ (D). HE: hematoxylin and eosin.

transitional meningiomas frequently carry NF2 mutations. ${ }^{1)}$ The results of our genetic analyses also indicated that the tumor in this case was different from transitional meningiomas. The term "dedifferentiated meningioma" was recently proposed for the existence of anaplastic and low-grade components in a single meningeal tumor. ${ }^{15,16)}$ In our case, both subtypes were low grade, not indicating dedifferentiated meningioma.

The present report illustrates a very rare condition in which two isolated components of fibrous meningioma coexisted within the main tumor component of meningothelial meningioma and showed the same molecular genetic profile, thus differing from transitional meningioma. This pathogenesis and the mechanisms involved are still unclear. One speculation is that while repeating cell division, the meningothelial cells might incidentally transform to the fibrous subtype and then forming the tumor mass. Another possibility is that the collagen fibers in meningothelial cells accumulate in isolation and form a tumor mass like fibrous meningioma due to environmental changes such as vascularity and oxygenation. Further advances in molecular genetic studies of this field might clarify the pathogenesis.

\section{Acknowledgments}

We express our sincere thanks to Dr. Yoshitaka Oda in the Department of Cancer Pathology at Hokkaido University School of Medicine for his technical support with molecular genetic analyses in this paper.

\section{Conflicts of Interest Disclosure}

None.

NMC Case Report Journal Vol.8, 2021 


\section{References}

1) Shibuya M: Pathology and molecular genetics of meningioma: recent advances. Neurol Med Chir (Tokyo) 55: 14-27, 2015

2) Clark VE, Erson-Omay EZ, Serin A, et al.: Genomic analysis of non-NF2 meningiomas reveals mutations in TRAF7, KLF4, AKT1, and SMO. Science 339: 1077-1080, 2013

3) Tsermoulas G, Turel MK, Wilcox JT, et al.: Management of multiple meningiomas. J Neurosurg 128: 1403-1409, 2018

4) Huang H, Buhl R, Hugo HH, Mehdorn HM: Clinical and histological features of multiple meningiomas compared with solitary meningiomas. Neurol Res 27: 324-332, 2005

5) Liu Y, Song DP, Wang T: Meningiomas with different histological grade in the same patient: case report. Medicine (Baltimore) 96: e9086, 2017

6) Mocker K, Holland H, Ahnert P, et al.: Multiple meningioma with different grades of malignancy: case report with genetic analysis applying single-nucleotide polymorphism array and classical cytogenetics. Pathol Res Pract 207: 67-72, 2011

7) Yuzawa S, Nishihara H, Yamaguchi S, et al.: Clinical impact of targeted amplicon sequencing for meningioma as a practical clinical-sequencing system. Mod Pathol 29: 708-716, 2016

8) Nakasu S, Hirano A, Shimura T, Llena JF: Incidental meningiomas in autopsy study. Surg Neurol 27: 319-322, 1987

9) Neuss M, Westphal M, Hänsel M, Herrmann HD: Clinical and laboratory findings in patients with multiple meningiomas. Br J Neurosurg 2: 249-256, 1988
10) Larson JJ, Tew JM, Simon M, Menon AG: Evidence for clonal spread in the development of multiple meningiomas. J Neurosurg 83: 705-709, 1995

11) Yan H, Luo K, Liu B, Kang J: A solitary fibrous tumor with concurrent meningioma at the same site: A case report and review of the literature. Oncol Lett 11: 3655-3659, 2016

12) Sayegh ET, Burch EA, Henderson GA, Oh T, Bloch O, Parsa AT: Tumor-to-tumor metastasis: breast carcinoma to meningioma. J Clin Neurosci 22: 268274, 2015

13) Brastianos PK, Horowitz PM, Santagata S, et al.: Genomic sequencing of meningiomas identifies oncogenic SMO and AKT1 mutations. Nat Genet 45: 285-289, 2013

14) Mirian C, Duun-Henriksen AK, Juratli T, et al.: Poor prognosis associated with TERT gene alterations in meningioma is independent of the WHO classification: an individual patient data meta-analysis. $J \mathrm{Neu}-$ rol Neurosurg Psychiatry 91: 378-387, 2020

15) Abedalthagafi MS, Bi WL, Merrill PH, et al.: ARID1A and TERT promoter mutations in dedifferentiated meningioma. Cancer Genet 208: 345-350, 2015

16) Shintaku M, Adachi Y, Arai A, Koyama J: Anaplastic and meningothelial meningiomas in a single tumor: A "dedifferentiated meningioma"? Neuropathology 36: 584-590, 2016

Corresponding author: Katsumi Sakata, MD

Department of Neurosurgery, Yokohama City University Medical Center, 4-57 Urafunecho, Minami-ku, Yokohama, Kanagawa 232-0024, Japan. e-mail:ksakata@med.yokohama-cu.ac.jp 